\title{
OPEN Diagnostic accuracy of ultrasound-guided core needle biopsy versus incisional biopsy in soft tissue sarcoma: an institutional experience
}

\author{
Miroslava Cernakova ${ }^{1}$, Gerhard M. Hobusch ${ }^{1 凶}$, Gabriele Amann ${ }^{2}$, Philipp T. Funovics ${ }^{1}$, \\ Reinhard Windhager ${ }^{1}$ \& Joannis Panotopoulos ${ }^{1}$
}

Core needle biopsy (CNB) is gaining in importance due to its advantages in the matter of patient morbidity, time and cost. Nevertheless, controversies still exist regarding the biopsy technique of choice for the accurate diagnosis of soft tissue sarcoma (STS). This retrospective cohort study compared the diagnostic performance between ultrasound-guided CNB and incisional biopsy (IB), both performed by orthopedic surgeons. The aims of the study were to answer the following questions: (1) Is ultrasound-guided CNB a highly reliable modality for diagnosing STSs? (2) Is CNB equally useful to IB for identifying histologic subtype? (3) Had patients who underwent CNB a reduced risk of complications? One-hundred and fifty-three patients who underwent resection of soft tissue sarcoma were classified into two groups according to biopsy technique prior to surgery; CNB group $(n=95)$ and IB group $(n=58)$. The final surgical specimens were in 40 patients liposarcoma (myxoid, pleomorphic and dedifferentiated), 39 undifferentiated pleomorphic sarcoma (UPS), 33 myxofibrosarcoma, 10 synovial sarcoma, 10 leiomyosarcoma and in the remaining 21 patients different soft tissue sarcoma entities. Sarcoma location of 71 patients was in the thigh, 19 in the lower leg, 22 in the upper arm and shoulder area; 10 in the knee and gluteal region, 9 in the thoracic region, the residual 12 in other body areas. Malignancy was correctly diagnosed in $87 \%$ ( 83 of 95 ) for the CNB group and $93 \%$ (54/58) for the IB group. Correct identification rate of histologic subtype was $80 \%$ (76 of 95 ) in the CNB group and $83 \%$ ( 48 of 58 ) in the IB group. There were no significant differences in the correct diagnosis rates of malignancy and subtype between the two techniques. No complications were seen in the CNB group, whereas 2 patients in whom IB was performed developed pulmonary embolism and 1 patient surgical site infection. Ultrasound-guided CNB is highly accurate and not inferior to IB in diagnosing the dignity of lesions and histologic subtype in patients with suspected STSs.

Soft tissue sarcomas (STS) represent a very heterogeneous group of malignant mesenchymal neoplasms with more than 50 histological subtypes ${ }^{1}$. While STS account for roughly only $1 \%$ of all adult malignancies ${ }^{2}$, there are even so over 27,000 new cases per year in EU27 countries ${ }^{3}$. Most STSs present in the extremities. The overall frequency of locations is thigh, buttock, and groin, $46 \%$; torso, $18 \%$; upper extremity, $13 \%$; retroperitoneal, $13 \%$; and head and neck, $9 \%{ }^{4}$. Diagnosis is often delayed by the fact that STSs can grow to a large extent until they cause discomfort and lead to consultation, especially in the thigh or pelvic regions $s^{5,6}$.

"Even for clinically unequivocal sarcomas, the importance of the preoperative histological diagnosis has been increasingly emphasized as different therapeutic regimes have been established for different sarcoma types"7. The standard diagnostic approach is a core needle biopsy or an open incisional biopsy except for superficial lesions $<$ $3 \mathrm{~cm}$ and the acral areas, which might be considered for excisional biopsies. Although current clinical guidelines

${ }^{1}$ Department of Orthopaedic Surgery, Medical University of Vienna, Vienna General Hospital, Währinger Gürtel 18-20, 1090 Vienna, Austria. ${ }^{2}$ Clinical Institute for Pathology, Medical University of Vienna, Vienna, Austria. ${ }^{\square}$ email: gerhard.hobusch@meduniwien.ac.at 


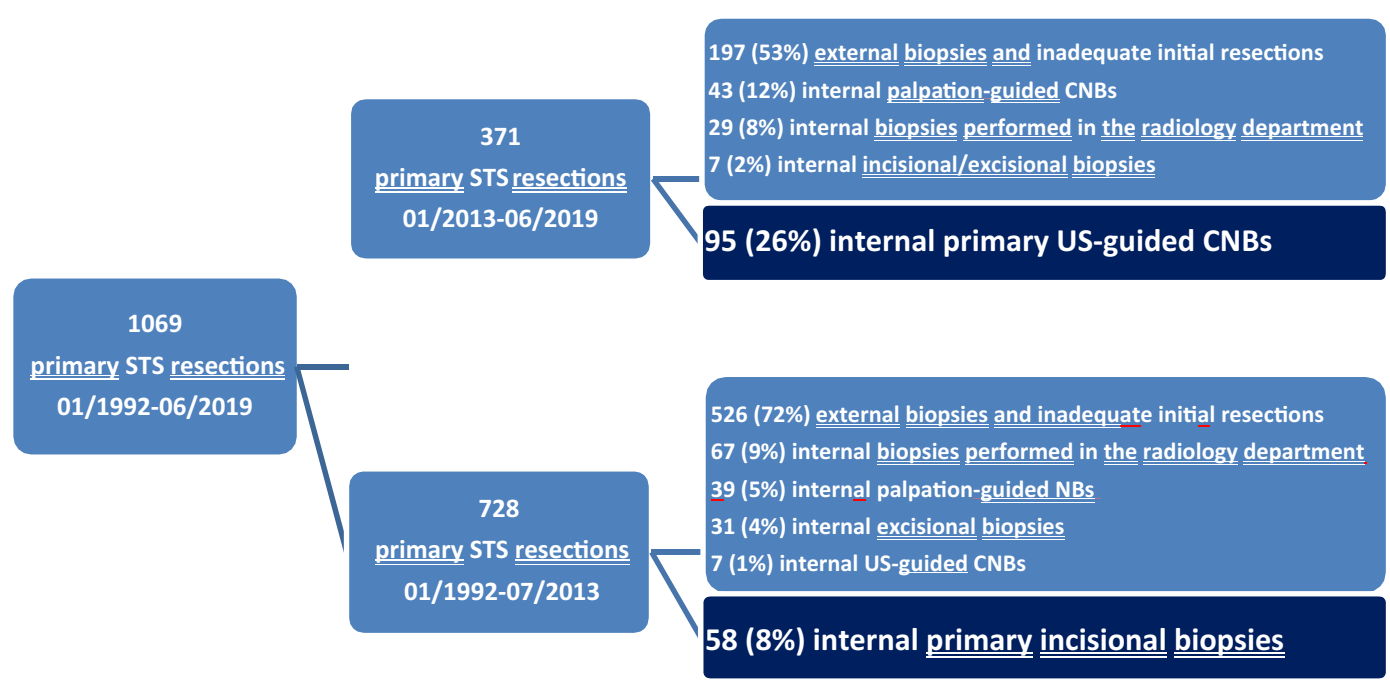

Figure 1. Elimination of patients.

indicate $\mathrm{CNB}$ to be the preferred technique over IB, disagreements still exist among clinicians and pathologists regarding the diagnostic accuracy of $\mathrm{CNB}^{8-11}$.

CNB has several advantages over open biopsies-in short time for diagnosis, in cost-effectiveness, and in number of patient visits necessary. The most often cited limitations of CNB are the ability to access deep-seated masses and the limited amount of sample material ${ }^{12,13}$. The open biopsy has been considered the gold standard for the diagnosis of STSs. However, IB frequently resulted in wound complications, tumor spreading, and inappropriate incisions, even making following surgical resection more difficult ${ }^{14,15}$.

\section{Patients and methods}

The study was approved by the ethics committee of the Medical University of Vienna, Vienna, Austria (approval number 1345/2020) in accordance with the Helsinki Declaration.

Given the retrospective design of this study, the ethics committee of the Medical University of Vienna waived the requirement for individual informed consent.

Patient selection. From our institution's tumor registry, patients who underwent soft tissue sarcoma resection between 01/1992 and 06/2019 were reviewed and classified into two groups according to primary in house biopsy techniques (CNB $n=95, I B n=58$ ) (Fig. 1). Open biopsy used to be the standard for evaluation of soft tissue tumors for many years. Nevertheless, since 2013 there has been a clear shift in our center towards CNB as the primary biopsy technique. IB has rather been reserved for special indications and cases of insufficient sampling by CNB. Excluded from this study were any cases of bony tissues, well differentiated liposarcoma, subsequent CNBs and IBs, all excisional biopsies, ultrasound-guided CNBs performed in the radiology department and CNBs not performed under US-guidance.

CNB group characteristics. The institution's tumor registry provided 95 patients ( $\mathrm{m} 47, \mathrm{f} 48$; average age at the biopsy: 60 [range 12-87] years) who had between $01 / 2013$ and $06 / 2019$ been treated by soft tissue sarcoma resection and in whom primary ultrasound-guided CNBs were performed at the Department of Orthopedics to diagnose the tumors. In twenty-four patients the final surgical specimens were liposarcoma (17 myxoid, 4 pleomorphic and 3 dedifferentiated), 30 myxofibrosarcoma, 21 undifferentiated pleomorphic sarcoma, 6 synovial sarcoma, 6 leiomyosarcoma and in the remaining 8 patients different STS entities. Sarcoma location of 45 patients was in the thigh, 9 in the lower leg, 9 in the shoulder area, 9 in the upper arm, 9 in the gluteal region, 5 in the thoracic region and the remaining 9 in different body regions (Table 1).

IB group characteristics. Fifty-eight patients (m 34, f 24; average age at the biopsy: 44[range 13-83] years) who between 01/1992 and 07/2013 underwent soft tissue sarcoma resection and in whom primary incisional biopsy was performed were identified. The final surgical specimens in 16 patients were liposarcoma (14 myxoid and 2 pleomorphic), 18 undifferentiated pleomorphic sarcoma, 4 synovial sarcoma, 4 leiomyosarcoma, 4 fibrosarcoma and in the remaining 12 cases different STS entities. Twenty-six STSs were located in the thigh, 10 in the lower leg, 8 in the knee area, 4 in the torso, 3 in the forearm and the remaining 7 in different body areas (Table 1).

Methods. Thorough reviews of medical records including the biopsy, histology and operative reports were performed, and the biopsy results compared with the final surgical specimens. Histological results are based on the pathology reports by experienced bone and soft tissue tumor pathologists at the institution for all patients included in this study. 


\begin{tabular}{|c|c|c|c|c|}
\hline & Final specimens CNB group & CNB matches & Final specimens IB group & IB matches \\
\hline Number of patients & 95 & $83(87 \%)$ & 58 & $54(93 \%)$ \\
\hline \multicolumn{5}{|l|}{ Histologic types } \\
\hline $\begin{array}{l}\text { Myxoid, pleomorphic and dedifferentiated } \\
\text { liposarcoma }\end{array}$ & 24 & $22(92 \%)$ & 16 & $15(94 \%)$ \\
\hline Undifferentiated pleomorphic sarcoma & 21 & $20(95 \%)$ & 18 & $17(94 \%)$ \\
\hline Myxofibrosarcoma & 30 & $24(80 \%)$ & 3 & 3 \\
\hline Synovial sarcoma & 6 & 6 & 4 & 4 \\
\hline Leiomyosarcoma & 6 & 4 & 4 & 4 \\
\hline Rhabdomyosarcoma & 3 & 3 & 3 & 3 \\
\hline (Myo)fibrosarcoma & 3 & 2 & 4 & 2 \\
\hline Epithelioid sarcoma & - & - & 3 & 3 \\
\hline Malignant peripheral nerve sheath tumor & 2 & 2 & - & - \\
\hline Clear cell sarcoma & - & - & 2 & 2 \\
\hline Alveolar soft part sarcoma & - & - & 1 & 1 \\
\hline \multicolumn{5}{|l|}{ Tumor location } \\
\hline Thigh & 45 & $38(84 \%)$ & 26 & $24(92 \%)$ \\
\hline Knee & 2 & 1 & 8 & 8 \\
\hline Lower leg & 9 & 8 & 10 & 10 \\
\hline Shoulder/axilla/scapula & 9 & 9 & 2 & 1 \\
\hline Upper arm & 9 & 9 & 2 & 2 \\
\hline Elbow & - & - & 1 & 1 \\
\hline Forearm & 2 & 2 & 3 & 3 \\
\hline Neck & 1 & 1 & - & - \\
\hline Torso & 5 & 4 & 4 & 4 \\
\hline Groin & 2 & 2 & 1 & - \\
\hline Iliac region & 2 & 1 & - & - \\
\hline Gluteal region & 9 & 8 & 1 & 1 \\
\hline
\end{tabular}

Table 1. Diagnostic accuracy in the assessment of dignity.

Statistical analysis. Statistical analyses were conducted with the Statistical Package for the Social Sciences (SPSS) for Windows (version 21; IBM Co., Endicott, NY, USA). Diagnostic accuracy was defined as the sum of true positive and true negative results divided by the total number of biopsies performed. The significance of differences between frequencies was calculated using Fisher's exact test. A p value of $<0.05$ was considered significant.

Ultrasound technique. Ultrasound was carried out using a GE Healthcare (GE Healthcare, Chicago, IL, USA) LogiqS8, software version Logiq S8: R1.5.4, linear probe:GE Healthcare ML6-15, 15.0Mhz. Needle biopsie was done under sterile conditions with or without coaxial needle with a semi-automatic biopsy needle G14 using "in-plane" technique. Usually, we took 3 to 4 samples.

\section{Results}

Diagnosing malignancy. CNB correctly identified 87\% (83/95) STSs (Table 1). Malignancy was, without any significant differences, reported by CNB in 92\% (22/24) liposarcoma, 80\% (24/30) myxofibrosarcoma and 95\% (20/21) undifferentiated pleomorphic sarcoma cases (Table 1). In three STSs (2\%; 2 myxofibrosarcoma and 1 myofibrosarcoma), the dignity was uncertain after CNB. Both myxofibrosarcoma as well as the myofibrosarcoma case were discussed in a multidisciplinary tumor board prior to surgery. Diagnostic accuracy of IB was 93\% (54/58). The dignity was uncertain in 2 STSs (3\%; 2 myofibrosarcoma), which were both resected due to radiologically suspected malignancy. There was no significant difference in correct malignancy diagnosis rate between the two techniques $(\mathrm{p}=0.29)$.

Identifying histologic subtype. Correct histologic subtype was by CNB reported in $80 \%(76 / 95)$ of the patients (Table 2). Correctly diagnosed were 80\% (24/30) myxofibrosarcoma, 90\% (19/21) UPS and 94\% (16/17) myxoid liposarcoma (Table 2). There were no significant differences found. Six STSs were by CNB misdiagnosed as other STS subtypes and in 1 patient the subtype could not be identified. Three pleomorphic liposarcoma, 1 dedifferentiated liposarcoma and 1 rhabdomyosarcoma were reported to be UPSs, 1 UPS to be an inflammatory fibrosarcoma and 1 malignant peripheral nerve sheath tumor (MPNST) could only be classified as a nonspecific malignant spindle cell lesion. IB identified correct histologic subtype in $83 \%(48 / 58)$ of the cases. Eighty-nine percent (16/18) UPS and 93\% (13/14) myxoid liposarcoma were correctly recognised. Misdiagnosed as other histologic subtype were 5 STSs, and in 1 case the subtype could not be identified. 


\begin{tabular}{|c|c|c|c|c|}
\hline & Final specimens CNB group & CNB matches & Final specimens IB group & IB matches \\
\hline Number of patients & 95 & $76(80 \%)$ & 58 & $48(83 \%)$ \\
\hline \multicolumn{5}{|l|}{ Histologic types } \\
\hline Undifferentiated pleomorphic sarcoma & 21 & $19(90 \%)$ & 18 & $16(89 \%)$ \\
\hline Myxofibrosarcoma & 30 & $24(80 \%)$ & 3 & 1 \\
\hline Myxoid liposarcoma & 17 & $16(94 \%)$ & 14 & $13(93 \%)$ \\
\hline Synovial sarcoma & 6 & 6 & 4 & 3 \\
\hline (Myo)fibrosarcoma & 3 & 2 & 4 & 1 \\
\hline Leiomyosarcoma & 6 & 4 & 4 & 4 \\
\hline Pleomorphic liposarcoma & 4 & 1 & 2 & 2 \\
\hline Rhabdomyosarcoma & 3 & 2 & 3 & 2 \\
\hline Dedifferentiated liposarcoma & 3 & 1 & - & - \\
\hline Epithelioid sarcoma & - & - & 3 & 3 \\
\hline Malignant peripheral nerve sheath tumor & 2 & 1 & - & - \\
\hline Clear cell sarcoma & - & - & 2 & 2 \\
\hline Alveolar soft part sarcoma & - & - & 1 & 1 \\
\hline
\end{tabular}

Table 2. Diagnostic accuracy in the assessment of histologic subtype.

One myxofibrosarcoma was classified as UPS, 1 synovial sarcoma as MPNST, 1 myxofibrosarcoma as myxoid liposarcoma, 1 UPS as leiomyosarcoma, 1 fibrosarcoma as synovial sarcoma and 1 rhabdomyosarcoma could only be classified as a highly malignant sarcoma. No significant difference in correct histologic subtype identification was found between CNB and IB ( $\mathrm{p}=0.83)$.

Grading. An examination of preoperative and postoperative grading concordance was only significant in CNB myxofibrosarcoma cases. Postoperative histological grades were reported in 22 by CNB as myxofibrosarcoma recognised cases. Additionally to histologic subtype, the correct grade was by CNB identified in $73 \%$ $(16 / 22)$ of myxofibrosarcoma.

Nondiagnostic samples. There were not significantly more nondiagnostic samples in the CNB group than the IB group. The rate of nondiagnostic samples was $9.5 \%(9 / 95)$ in the CNB group and $3 \%(2 / 58)$ in the IB group. There were six samples with necrotic tissue, 2 samples with skeletal muscle and 1 sample with insufficient material in the CNB group. In the IB group was 1 sample with suspected reactive lesion and 1 sample with mostly thrombus material.

Complications. Patients who underwent CNB showed no complications. In the IB group, 1 male patient (risk factors for a pulmonary embolism: 1. cancer, 2. recent surgery) developed a pulmonary embolism 9 days and 1 female patient (risk factors for pulmonary embolism: 1. cancer, 2. recent surgery, 3. venous insufficiency, 4. smoking, 5. increasing age) 6 days after the surgical procedure. Surgical site infection occurred in 1 patient in whom IB was performed.

The difference in the complication rates between the two techniques was not significant.

\section{Discussion}

"In light of the growing understanding of different biologies and sensitivities of the various STS subtypes, the value of histology in the selection of peri-operative treatments is one of the most intriguing topics under discussion ${ }^{16}$." In this perspective, discussions about the ideal biopsy techniques are still ongoing. To help solve this diagnostic dilemma, we performed an institutional study regarding the diagnostic performance of CNB and IB in STS. Our results indicate that ultrasound-guided CNB is highly accurate and not inferior to IB in diagnosing the dignity of lesions and histologic subtype in patients with suspected STSs.

The ideal biopsy technique should be characterized by a high diagnostic accuracy, and be simple while minimizing morbidity, limiting potential tumor spread, and avoiding interference with future treatments $\mathrm{s}^{8,12}$. Siddiqi et al. reported no definable adverse effect on local recurrence is associated with not resecting the CNB tract during definitive operation.

Any influence of a CNB tract resection is likely to be of minor clinical importance ${ }^{17}$. A recent meta-analysis demonstrates fewer complications compared with $\mathrm{IB}^{8}$. Similarly, in this study, no complications were seen in patients in whom CNB was performed, whereas pulmonary embolisms occurred in 2 patients who underwent IB. Sarcoma alone is a provoking factor for a pulmonary embolism, recent surgical procedures increase the risk even more ${ }^{18}$. The mobility after incisional biopsies is mostly reduced up to two weeks, whereas there are no limitations after CNB.

The study has several limitations. Our examination of diagnostic accuracy in terms of grading of STSs was limited. Nevertheless, our results were in accordance with the known fact that current grading is not ideal for CNBs. CNB is prone to sampling error with the risk of underestimation of grade ${ }^{19,20}$, and new developments in 
molecular genetics and targeted therapies question the future of classical grading. It could eventually be replaced by molecular parameters and biomarkers ${ }^{19,21}$.

However, histologic grade is important prognostic factor and indicator of metastatic risk for STSs. Therefore, it should be part of the pathology report and complemented with radiologic parameters when dealing with a needle biopsy ${ }^{19}$.

More than one pathologist was involved in this study and more than one orthopedic surgeon performed the biopsies. The high IB and ultrasound-guided CNB diagnostic performances in our sarcoma center might well be the result of an established workflow between experienced surgeons, radiologists and dedicated sarcoma pathologists. However, without this multidisciplinary setting, there are still numerous cases with incorrect and unplanned open biopsies as the primary biopsy technique performed outside of sarcoma centers. The retrospective data outlined the clear shift in our center toward CNB.

Likewise our study, a meta-analysis published in 2020 reports high CNB accuracy in diagnosing the dignity of lesions and STS histotype in patients with suspected STS ${ }^{8}$. However, two other recent meta-analyses demonstrate a higher diagnostic accuracy for IB compared with $\mathrm{CNB}^{10,11}$. The CNB-to-IB ratio was 1.6:1.0 in this study compared to 3-10:1 in the 8 centers included in the meta-analysis carried out by Birgin et al. Moreover, there were no different biopsy techniques and imaging modalities used in the CNB group. All CNBs were performed under US-guidance with a semi-automatic G14 biopsy needle using "in-plane" technique. Any cases of bony tissues were excluded from this study. All included CNBs and IBs were the primary biopsies, all sequential biopsies were excluded from this study.

\section{Conclusion}

Considering the high diagnostic accuracy rates of both techniques and a significantly higher invasiveness of IB, we recommend ultrasound-guided CNB as the primary technique in patients with suspected STSs. There is always the option to perform IB after CNB in cases of insufficient sampling, but this is not consistently true the other way around. At least 14-gauge needles should be used. To access deep-seated masses in the pelvic region, primary CT- and MRT-guidance should be considered for CNBs.

Received: 20 April 2021; Accepted: 9 August 2021

Published online: 08 September 2021

\section{References}

1. Fletcher, C. D. M. The evolving classification of soft tissue tumours: An update based on the new 2013 WHO classification. Histopathology 64(1), 2-11 (2014).

2. Jemal, A. et al. Cancer statistics, 2007. CA Cancer J. Clin. 57(1), 43-66 (2007).

3. Stiller, C. A. et al. Descriptive epidemiology of sarcomas in Europe: Report from the RARECARE project. Eur. J. Cancer. 49(3), 684-695 (2013).

4. Nedea, E. A. \& DeLaney, T. F. Sarcoma and skin radiation oncology. Hematol. Oncol. Clin. N. Am. 20(2), 401-429 (2006).

5. Panotopoulos, J., Funovics, P. \& Windhager, R. Weichteilsarkome [Soft tissue sarcoma]. Z. Orthop. Unfall. 152(3), 277-291 (2014).

6. Windhager, R. \& Hobusch, G. M. The role of surgery in soft tissue sarcoma: Can we improve outcome and function towards sporting activities?. Mag. Eur. Med. Oncol. 13, 164-168 (2020).

7. Agaimy, A. Value of core needle biopsy in preoperative diagnostics of soft tissue tumors. Possibilities and limitations. Pathologe. 35, 189-197 (2014).

8. Birgin, E. et al. Core needle biopsy versus incisional biopsy for differentiation of soft-tissue sarcomas: A systemic review and meta-analysis. Cancer 126(9), 1917-1928 (2020).

9. Errani, C., Traina, F., Perna, F., Calamelli, C. \& Faldini, C. Current concepts in the biopsy of musculoskeletal tumors. Sci. World J. 2013, 538152 (2013).

10. Kubo, T. et al. A meta-analysis supports core needle biopsy by radiologists for better histological diagnosis in soft tissue and bone sarcomas. Medicine 97, e11567 (2018).

11. Traina, F. et al. Current Concepts in the biopsy of musculoskeletal tumors. J. Bone Joint Surg. Am. 97, e7 (2015).

12. Kasraeian, S., Allison, D. C., Ahlmann, E. R., Fedenko, A. N. \& Menendez, L. R. A comparison of fine-needle aspiration, core biopsy, and surgical biopsy in the diagnosis of extremity soft tissue masses. Clin. Orthop. Relat. Res. 468, 2992-3002 (2010).

13. Kilpatrick, S. E., Cappellari, J. O., Bos, G. D., Gold, S. H. \& Ward, W. G. Is fine-needle aspiration biopsy a practical alternative to open biopsy for the primary diagnosis of sarcoma? Experience with 140 patients. Am. J. Clin. Pathol. 115(1), 59-68 (2001).

14. Mankin, H. J., Lange, T. A. \& Spanier, S. S. The hazards of biopsy in patients with malignant primary bone and soft-tissue tumors. J. Bone Joint Surg. Am. 64(8), 1121-1127 (1982).

15. Springfield, D. S. \& Rosenberg, A. Biopsy: Complicated and risky. J. Bone Joint Surg. Am. 78(5), 639-643 (1996).

16. Saponara, M., Stacchiotti, S., Casali, P. G. \& Gronchi, A. (Neo)adjuvant treatment in localized soft tissue sarcoma: Un unsolved affair. Eur. J. Cancer. 70, 1-11 (2017).

17. Siddiqi, M. A., Kim, H. S., Jede, F. \& Han, I. Association of core needle biopsy tract resection with local recurrence in extremity soft tissue sarcoma. Skeletal Radiol. 46(4), 507-512 (2017).

18. Corrigan, D., Prucnal, C. \& Kabrhel, C. Pulmonary embolism: The diagnosis, risk-stratification, treatment and disposition of emergency department patients. Clin. Exp. Emerg. Med. 3(3), 117-125 (2016).

19. Coindre, J. M. Grading of soft tissue sarcomas: Review and update. Arch. Pathol. Lab. Med. 130(10), 1448-1453 (2006).

20. Ray-Coquard, I. et al. Evaluation of core needle biopsy as a substitute to open biopsy in the diagnosis of soft tissue masses. Eur. J. Cancer. 39(14), 2021-2025 (2003).

21. Panotopoulos, J. et al. Hemoglobin, alkalic phosphatase, and C-reactive protein predict the outcome in patients with liposarcoma. J. Orthop. Res. 33(5), 765-770 (2015).

\section{Author contributions}

M.C. and G.M.H. designed research and wrote the manuscript. G.M.H., G.A., P.T.F., R.W. and J.P. substantively revised the work. 


\section{Competing interests}

The authors declare no competing interests.

\section{Additional information}

Correspondence and requests for materials should be addressed to G.M.H.

Reprints and permissions information is available at www.nature.com/reprints.

Publisher's note Springer Nature remains neutral with regard to jurisdictional claims in published maps and institutional affiliations.

(c) (1) Open Access This article is licensed under a Creative Commons Attribution 4.0 International License, which permits use, sharing, adaptation, distribution and reproduction in any medium or format, as long as you give appropriate credit to the original author(s) and the source, provide a link to the Creative Commons licence, and indicate if changes were made. The images or other third party material in this article are included in the article's Creative Commons licence, unless indicated otherwise in a credit line to the material. If material is not included in the article's Creative Commons licence and your intended use is not permitted by statutory regulation or exceeds the permitted use, you will need to obtain permission directly from the copyright holder. To view a copy of this licence, visit http://creativecommons.org/licenses/by/4.0/.

(C) The Author(s) 2021 